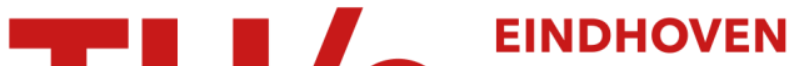 \\ UNIVERSITY OF \\ TECHNOLOGY
}

\section{Iterative detection for pretransformed OFDM by subcarrier reconstruction}

Citation for published version (APA):

Ho, C. K., Lei, Z., Sun, S., \& Wu, Y. (2005). Iterative detection for pretransformed OFDM by subcarrier reconstruction. IEEE Transactions on Signal Processing, 53(8), 2842-2854.

https://doi.org/10.1109/TSP.2005.850371

DOI:

10.1109/TSP.2005.850371

Document status and date:

Published: 01/01/2005

\section{Document Version:}

Publisher's PDF, also known as Version of Record (includes final page, issue and volume numbers)

\section{Please check the document version of this publication:}

- A submitted manuscript is the version of the article upon submission and before peer-review. There can be important differences between the submitted version and the official published version of record. People interested in the research are advised to contact the author for the final version of the publication, or visit the $\mathrm{DOI}$ to the publisher's website.

- The final author version and the galley proof are versions of the publication after peer review.

- The final published version features the final layout of the paper including the volume, issue and page numbers.

Link to publication

\section{General rights}

Copyright and moral rights for the publications made accessible in the public portal are retained by the authors and/or other copyright owners and it is a condition of accessing publications that users recognise and abide by the legal requirements associated with these rights.

- Users may download and print one copy of any publication from the public portal for the purpose of private study or research.

- You may not further distribute the material or use it for any profit-making activity or commercial gain

- You may freely distribute the URL identifying the publication in the public portal.

If the publication is distributed under the terms of Article 25fa of the Dutch Copyright Act, indicated by the "Taverne" license above, please follow below link for the End User Agreement:

www.tue.nl/taverne

Take down policy

If you believe that this document breaches copyright please contact us at:

openaccess@tue.nl

providing details and we will investigate your claim. 


\title{
Iterative Detection for Pretransformed OFDM by Subcarrier Reconstruction
}

\author{
Chin Keong Ho, Student Member, IEEE, Zhongding Lei, Senior Member, IEEE, Sumei Sun, and Wu Yan
}

\begin{abstract}
In this paper, an iterative detection method for an uncoded pretransformed (PT) orthogonal frequency division multiplexing (OFDM) system where the channel is not known at the transmitter is proposed. The iterative detection starts with linear detection. The noiseless received signal at the weakest subcarrier (corresponding to the smallest channel amplitude) is estimated based on all the detected data symbols using a hard or soft decision. Then, the actual received signal at the weakest subcarrier is replaced by the estimated one. This process is referred to as reconstruction here. After reconstruction, linear detection is carried out again to generate the next set of symbol estimates. The whole process proceeds iteratively to reconstruct the received signal at the next-weakest subcarrier. The transform coefficients and the iterative process are designed to maximize the minimum signal-to-noise power ratio. Under the assumption that the previous detection is error free, it is shown analytically that the iterative method achieves a diversity advantage of $i+1$ in the $i$ th iteration, thus providing an explanation of its superior performance. Due to the flexibility of the transform design, the analysis conducted is applicable for other common systems as well. Simulations in realistic channels are carried out, and the bit-error rate performance of the iterative detection is superior as compared to that of the conventional detectors for the PT-OFDM or OFDM system.
\end{abstract}

Index Terms-Iterative detection, linear detection, MC-CDMA, OFDM, OFDM-CDMA, pretransformed OFDM, SC-FDE, spread OFDM, transform design, WHT-OFDM.

\section{INTRODUCTION}

$\mathbf{F}$ UTURE wireless systems have to be highly spectral efficient to achieve high user capacities and high data rates. Multicarrier modulation realized by orthogonal frequency division multiplexing (OFDM) [1], [2] is well suited for high data rate applications in fading channels and has been chosen for several standards, such as digital audio broadcasting (DAB) [3], digital video broadcasting (DVB-T) [4], and three broadband wireless LAN standards: IEEE 802.11a, European HIPERLAN/2, and Japanese multimedia mobile access communication systems [5].

\footnotetext{
Manuscript received January 7, 2004; revised July 31, 2004. This paper was presented in part at the IEEE Vehicular Technology Conference, Jeju, Korea, April 2003 and the IEEE International Symposium on Personal, Indoor, and Mobile Radio Communications, Barcelona, Spain, September 2004. The associate editor coordinating the review of this manuscript and approving it for publication was Prof. Karim Drouiche.

C. K. Ho was with the Institute for Infocomm Research, Singapore 119613. $\mathrm{He}$ is now with the Department of Electrical Engineering, Eindhoven University of Technology, 5600 MB Eindhoven, The Netherlands (e-mail: c.k.ho@tue.nl).

Z. Lei, S. Sun, and W. Yan are with the Institute for Infocomm Research, Singapore 119613 (e-mail: leizd@i2r.a-star.edu.sg; sunsm@i2r.a-star.edu.sg; wuyan@i2r.a-star.edu.sg).

Digital Object Identifier 10.1109/TSP.2005.850371
}

An OFDM system transfers a frequency-selective fading channel to multiple flat-fading parallel transmission channels or subcarriers in the frequency domain by using a cyclic prefix (CP). The use of $\mathrm{CP}$ also removes the interblock interference and allows processing to be carried out in a block-by-block manner. In such a system, the information sent on some carriers might be subjected to strong attenuations and might not be recovered correctly at the receiver. This has motivated the proposal of more robust transmission schemes combining the advantages of code division multiple access (CDMA) [6] with the advantages of OFDM, in which the information is spread across all the subcarriers by some pretransformation (PT) matrix, referred to here in general as PT-OFDM. By using a different transform, PT-OFDM is also known as OFDM-CDMA [7], spread OFDM [8], WHT-OFDM [9], [10], or multicarrier (MC)-CDMA [11]. The PT-OFDM has been investigated in a European Information Society Technologies program, as a promising candidate for future wireless communications with a very high data rate transmission [9]. The single carrier frequency domain equalization (SC-FDE) [12], [13] system is also a special case of a PT-OFDM system, where the pretransformation is equal to the Fourier transform. It has been proposed to the IEEE 802.16 working group and as a possible solution for a broadband wireless metropolitan area network system [13].

The PT-OFDM system falls under a broad class of linear block-based transmission schemes [14]. A general treatment of the linear block system where the interblock interference can be removed via the use of zero padding or CP is given in [14] and [15]. System designs optimized with respect to signal-to-noise power ratio (SNR) or bit-error rate (BER) are derived in [14] and [15], respectively; however, knowledge of the channel is required at the transmitter.

In [16], where the CP is used, as in the PT-OFDM system considered here, no channel knowledge is required at the transmitter. The transform used for the PT-OFDM system is optimized to minimize the BER when a linear least-squares (LS) or minimum mean-squared error (MMSE) detector is used. The BER could be further improved since the diversity gain that could be exploited is limited through the use of linear detectors. In [17] and [18], the diversity advantage is fully exploited when a maximum-likelihood (ML) detector is used. However, high detection complexity, which increases exponentially with the size of the transform, is required. Although it is demonstrated in [18] that superior performance can be obtained if linear detectors are used for detecting binary phase shift keying (BPSK) modulated symbols using the same transform design, the per- 
formance is not as promising when complex symbols are used. In [19] and [20], an iterative detector based on parallel interference cancellation (PIC) is proposed for multiuser detection. In the context of PT-OFDM, the number of users and the transform size is equal and is usually large. The PIC when applied to the PT-OFDM system will result in a high complexity detector.

As far as transform design is concerned, optimality of a transform is subjected to the detector employed at the receiver. A transform designed to optimize the performance for the ML detector is proposed in [21]. Another related work [22] designs the transforms to be used in a novel manner. A BPSK data stream is split into two data streams and transmitted concurrently via different transforms (akin to different codes in a multiuser case) in an OFDM system. The orthogonal transforms are designed to minimize the intercode interference. Similarly, when complex symbols are used instead, obtaining a superior performance is not straightforward. When the transform is selected randomly, [23] has investigated the asymptotic performance of a PT-OFDM system when the transform size (and channel diversity) goes to infinity.

The BER is usually the most important performance measure. However, the transform in PT-OFDM can be designed to cater specifically for other attractive properties as well, such as lower clipping probability, less spectral regrowth, and lower block error rate, as explored in [9], [24], and [25]. These could be used as secondary optimizing objectives when a sufficient degree of freedom is available in the transform design.

The main contributions of this paper are as follows.

- We introduce a novel low-complexity iterative detector that improves the BER performance at a very low computational cost. The iterative detection starts with a linear LS or MMSE detector. The received signal at a certain subcarrier index is replaced by an estimated one based on information of all the previously detected symbols. We called this process reconstruction. The process of data detection and reconstruction is carried out iteratively.

- We provide analysis on the optimization of the transform coefficients and reconstruction method to maximize the minimum SNR. A class of unitary matrices with constant magnitude satisfies the transform design requirements, and its large degree of freedom allows one the flexibility of choosing the transforms to satisfy some secondary objectives as well. Furthermore, reconstruction should be carried out starting from the subcarrier with the smallest channel amplitude (we called this the weakest subcarrier). In the next iteration, the next-weakest subcarrier is selected for reconstruction, and so on.

- We carried out the performance analysis of the proposed iterative detector by bounding the SNR appropriately. We make the ideal assumption that the previous detections are error free. Our main result is that, for an identical independent Rayleigh distributed channel of each subcarrier, the iterative method achieves a diversity advantage of $i+1$ in the $i$ th iteration. The simulation results, in realistic channels, illustrate that the BER performance of the iterative detection is much better than that of the conventional detectors for PT-OFDM or OFDM system.
It should be noted that this iterative detector differs from the algorithms commonly used in the multiuser detection context, such as PIC. The conventional iterative process proceeds user by user (corresponding to data symbol by symbol here) to cancel multiuser/intersymbol interference. Our method involves a reconstruction and detection process instead, subcarrier by subcarrier. Theoretically, if the complexity allows, these two iterative detection methods may be implemented together. It should also be noted that this subcarrier-by-subcarrier-based iterative detection method does not apply to the conventional OFDM system, since data symbols in a conventional OFDM system are transmitted independently on orthogonal subcarriers. It is the presence of the spreading over the data symbols on all subcarriers that allows the iterative algorithm to kick off.

This paper is organized as follows. First, the PT-OFDM system model is described in Section II. The iterative detection method is given in Section III, whereby the transform coefficients and reconstruction method are derived to maximize the minimum SNR across different symbols. The flexibility of the transform design is explored, and refinements of the algorithm are suggested in order to realize better performance. In Section IV, by assuming perfect reconstruction, we derive the upper and lower bounds of the instantaneous SNR. By using order statistics, the lower and upper bounds of the BER based on the iterative detector are obtained. A comparison of the analytical performance of the PT-OFDM system with and without iterations is also presented. Finally, simulations are conducted in Section VI to illustrate the performance of the proposed detector, and the conclusion is given in Section VII.

Throughout this paper, the following notations are adhered to. Bold lowercase letters are used to denote column vectors. Bold uppercase letters are used to denote matrices. The superscripts ${ }^{*}, T$, and ${ }^{H}$ are used to denote complex conjugate, transpose, and Hermitian, respectively. The $(m, n)$ th element of matrix $\mathbf{W}$ and the $m$ th element of vector $\mathbf{w}$ are denoted as $w_{m n}$ and $w_{m}$, respectively. The identity matrix is denoted as $\mathbf{I}$.

\section{SYSTEM DESCRIPTION}

\section{A. PT-OFDM}

The PT-OFDM system block is depicted in Fig. 1. For simplicity, we consider a system with $M=2^{k}$ subcarriers for some positive integer $k$, and $M$ information symbols $x_{m}$, $m=1,2, \cdots M$ are transmitted at the same time in one OFDM symbol. The modulation symbols $s_{m}$ of the subcarriers are calculated from the information symbols $x_{m}$ using the matrix operation

$$
\mathbf{s}=\mathbf{W} \cdot \mathbf{x}
$$

where $\mathbf{s}=\left[s_{1}, s_{2}, \ldots, s_{M}\right]^{T}, \mathbf{x}=\left[x_{1}, x_{2}, \ldots, x_{M}\right]^{T}$, and $\mathbf{W}$ represents the PT matrix of size $M \times M$. Therefore, there is no loss of code rate in terms of number of information symbols transmitted per channel use. In the case of an OFDM system, W is simply an identity matrix. The block of modulation symbols $\mathbf{s}$ is then passed through an inverse discrete Fourier transform, which can be implemented efficiently using the inverse fast Fourier transform (IFFT). After inserting a cyclic prefix with duration no shorter than the maximum channel delay spread, 


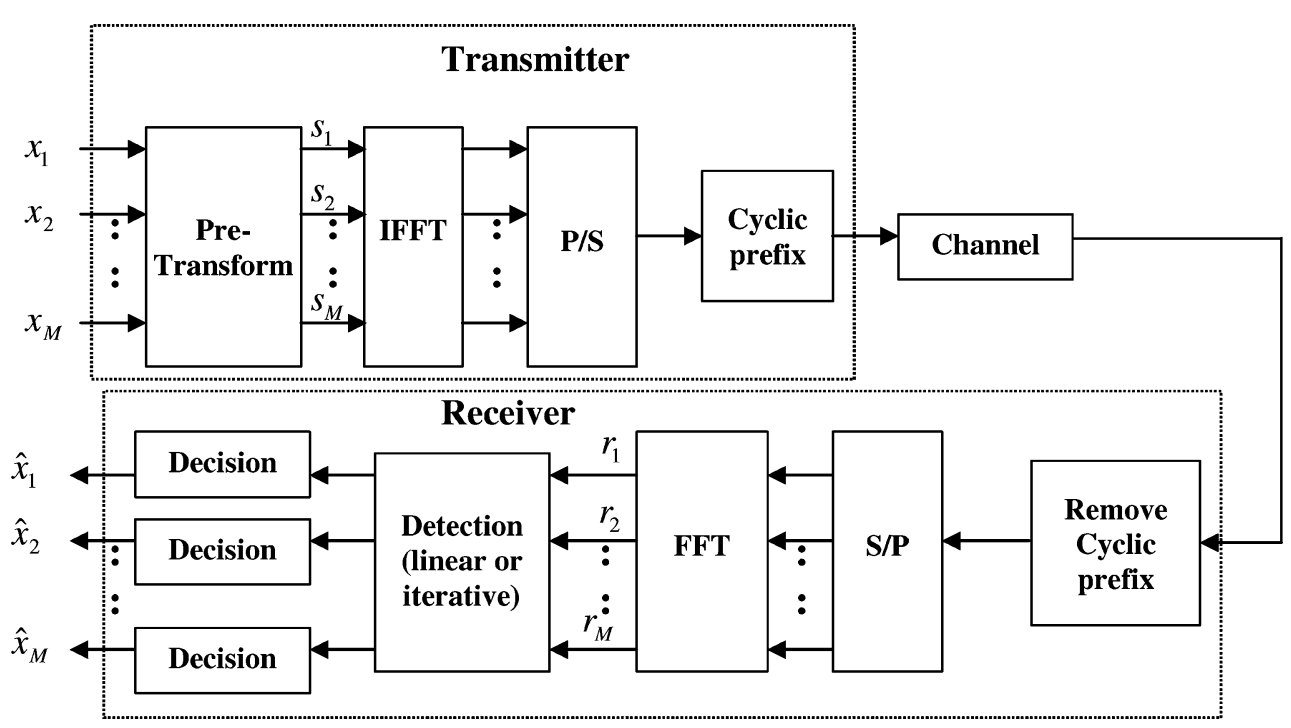

Fig. 1. PT-OFDM system block diagram.

the PT-OFDM symbol is transmitted. The channel is assumed to be a quasi-static frequency selective Rayleigh fading channel corrupted by additive white Gaussian noise (AWGN).

At the receiver, the samples of the received signal corresponding to the cyclic prefix are removed. The output vector after FFT $\mathbf{r}=\left[r_{1}, r_{2}, \ldots, r_{M}\right]^{T}$ can be written as

$$
\mathbf{r}=\boldsymbol{\Gamma} \cdot \mathbf{s}+\mathbf{n}=\boldsymbol{\Gamma} \cdot \mathbf{W} \cdot \mathbf{x}+\mathbf{n}
$$

where $\Gamma=\operatorname{diag}\left(h_{1}, h_{2}, \ldots, h_{M}\right)$, a diagonal matrix with diagonal elements $h_{1}, h_{2}, \ldots, h_{M}$, is the channel in the frequency domain, and $\mathbf{n}$ is the $M \times 1$ AWGN vector with variance $\sigma_{n}^{2}$. The frequency domain channel coefficient is given as $h_{m}=$ $\sum_{n} \tilde{h}_{n} \exp (-j 2 \pi n(m-1) / M), m=1,2, \cdots, M$, assuming a sampled spaced $L_{h}$ th-order FIR channel model $\left\{\tilde{h}_{n}\right\}_{n=0}^{L_{h}}$.

\section{Detection AlgorithMS}

The iterative detection algorithm consists of three stages for each iteration, namely, reconstruction, linear filtering, and decision. For clarity, we shall describe the simplest method to implement each stage and discuss the variation of each stage at the end of the section.

\section{A. Initialization}

The initial iteration skips the step of reconstruction but proceeds by removing the interference amongst different information symbols. We minimize the squared error after linear filtering by using the LS criteria. Thus, the received signal vector $\mathbf{r}$ has to be linearly filtered using

$$
\mathbf{G}=\mathbf{W}^{-1} \cdot \boldsymbol{\Gamma}^{-1}=\mathbf{W}^{-1} \cdot \operatorname{diag}\left(\frac{1}{h_{1}}, \frac{1}{h_{2}}, \ldots, \frac{1}{h_{M}}\right) \text {. }
$$

This is also known as the zero-forcing (ZF) or orthogonality restoring combining (ORC) detector in the MC-CDMA literature in a synchronous system [26]. As weak subcarriers are amplified with high gain, they introduce a high noise level.
Applying the LS filter $\mathbf{G}$ on the received signal $\mathbf{r}$ given by (2), we get

$$
\widetilde{\mathbf{x}}_{0} \triangleq \mathbf{G} \cdot \mathbf{r}=\mathbf{x}+\mathbf{W}^{-1} \Gamma^{-1} \mathbf{n}
$$

The $j$ th element of $\widetilde{\mathbf{x}}_{0}$, denoted as $\widetilde{x}_{0, j}$, can be written as

$$
\widetilde{x}_{0, j}=x_{j}+\sum_{m=1}^{M} \frac{\psi_{j m} n_{m}}{h_{m}}, \quad j=1,2, \cdots M
$$

where $\psi_{j m}$ is the $(j, m)$ th element of the matrix $\Psi \triangleq \mathbf{W}^{-1}$.

We then perform hard decision on the filtered signal to get

$$
\hat{\mathbf{x}}_{0}=\operatorname{dec}\left(\widetilde{\mathbf{x}}_{0}\right)
$$

where $\operatorname{dec}(\cdot)$ stands for the decision function. Denote $\hat{\mathbf{x}}_{0}=\mathbf{x}+$ $\mathbf{e}_{0}$, where $\mathbf{e}_{0}$ represents the error vector due to a wrong decision made. These procedures constitute the initial iteration (we refer to this as iteration $i=0$ ). If required, $\hat{\mathbf{x}}_{0}$ can be used to detect the transmitted signal. However, further iterations can result in better performance.

\section{B. Subsequent Iterations}

We denote the received signal vector when noise is absent as $\mathbf{r}_{\text {noiseless }}=\Gamma \mathbf{W} \mathbf{x}$. We can estimate this vector by using $\hat{\mathbf{x}}_{0}$ as follows:

$$
\widetilde{\mathbf{r}}_{1} \triangleq \Gamma \mathbf{W} \hat{\mathbf{x}}_{0}=\mathbf{r}_{\text {noiseless }}+\widetilde{\mathbf{e}}_{1}
$$

where $\widetilde{\mathbf{e}}_{1}=\boldsymbol{\Gamma} \mathbf{W} \mathbf{e}_{0}$ represents the (introduced) noise due to the error vector $\mathbf{e}_{0}$ in the decision. From this, we see that the reconstructed vector $\widetilde{\mathbf{r}}_{1}$ removes the noise from the receiver but introduces error due to the wrong detection in the previous iteration (i.e., trade $\widetilde{\mathbf{e}}_{1}$ with $\mathbf{n}$ ). This tradeoff is not worthwhile since the likelihood of a wrong symbol detection is high. Thus, it is probable that this will result in an even larger increase in interference due to error propagation rather than only the anticipated reduction of noise. 
Consider only substituting one element of $\mathbf{r}$, say, the $m_{1}$ th element, instead of the whole vector. We denote $\mathcal{I}_{m}$ as a diagonal matrix with value 1 on its $m$ th diagonal term and 0 otherwise and $\mathcal{E}_{m}$ as a diagonal matrix with value 0 on its $m$ th diagonal term and 1 otherwise. ${ }^{1}$ The proposed reconstructed vector is carried out as

$$
\begin{aligned}
\mathbf{r}_{1} & =\mathcal{E}_{m_{1}} \mathbf{r}_{0}+\mathcal{I}_{m_{1}} \widetilde{\mathbf{r}}_{1} \\
& =\mathbf{r}_{\text {noiseless }}+\mathcal{I}_{m_{1}} \widetilde{\mathbf{e}}_{1}+\mathcal{E}_{m_{1}} \mathbf{n}
\end{aligned}
$$

where we add the subscript 0 to the received vector to denote $\mathbf{r}_{0}=\mathbf{r}$, so that indices for subsequent iterations can be generalized easily. We defer how to maximize the benefit of this tradeoff by choosing $m_{1}$ appropriately to Section III-C.

For the first iteration $(i=1)$, before detection, we use the filter $\mathbf{G}$ to estimate $\mathbf{x}$ again but using $\mathbf{r}_{1}$

$$
\begin{aligned}
\widetilde{\mathbf{x}}_{1} & =\mathbf{G r}_{1} \\
& =\mathbf{x}+\mathbf{W}^{-1} \boldsymbol{\Gamma}^{-1} \mathcal{I}_{m_{1}} \widetilde{\mathbf{e}}_{1}+\mathbf{W}^{-1} \boldsymbol{\Gamma}^{-1} \mathcal{E}_{m_{1}} \mathbf{n} \\
& =\mathbf{x}+\mathbf{W}^{-1} \mathcal{I}_{m_{1}} \mathbf{W} \mathbf{e}_{0}+\mathbf{W}^{-1} \boldsymbol{\Gamma}^{-1} \mathcal{E}_{m_{1}} \mathbf{n}
\end{aligned}
$$

since $\Gamma^{-1} \mathcal{I}_{m_{1}}=\mathcal{I}_{m_{1}} \Gamma^{-1}$, as both are diagonal matrices. The $j$ th element of $\widetilde{\mathbf{x}}_{1}$ can be written as

$$
\widetilde{x}_{1, j}=x_{j}+v_{1, j}+\sum_{m=1, m \neq m_{1}}^{M} \frac{\psi_{j m} n_{m}}{h_{m}}, \quad j=1,2, \cdots M
$$

where $v_{1, j}$ is the $j$ th element of $\mathbf{v}_{1} \triangleq \mathbf{W}^{-1} \mathcal{I}_{m_{1}} \mathbf{W} \mathbf{e}_{0}$. A decision function would yield the hard decided output as

$$
\hat{\mathbf{x}}_{1}=\operatorname{dec}\left(\widetilde{\mathbf{x}}_{1}\right)
$$

hence completing the first iteration. For the second iteration and onwards, similar procedures are used for the first iteration.

We summarize the $i$ th iteration $i=1,2, \cdots$ as

- reconstruction: $\mathbf{r}_{i}=\mathcal{E}_{m_{i}} \mathbf{r}_{i-1}+\mathcal{I}_{m_{i}} \boldsymbol{\Gamma W} \hat{\mathbf{x}}_{i-1}$;

- filtering: $\widetilde{\mathbf{x}}_{i}=\mathbf{G r}_{i}$, where $\mathbf{G}=\mathbf{W}^{-1} \boldsymbol{\Gamma}^{-1}$;

- detection: $\hat{\mathbf{x}}_{i}=\operatorname{dec}\left(\widetilde{\mathbf{x}}_{i}\right)$.

We define a set containing all the indices that has been used for reconstruction as $\mathcal{M}_{i}=\left\{m_{1}, m_{2}, \cdots, m_{i}\right\}$. The complementary set of indices not used for reconstruction is denoted as $\mathcal{M}_{i}^{C}=\left\{m_{i+1}, m_{2}, \cdots, m_{M}\right\}$. In order to facilitate subsequent derivations, in iteration $i$, we generalize the $j$ th element of $\widetilde{\mathbf{x}}_{i}$ as

$$
\widetilde{x}_{i, j}=x_{j}+v_{i, j}+\sum_{m \in \mathcal{M}_{i}^{C}} \frac{\psi_{j m} n_{m}}{h_{m}}, \quad j=1,2, \cdots M .
$$

\section{Transform Design and Reconstruction Criteria}

At this point, we have left the questions of which transform to use and the choice of $\mathcal{M}_{i}$ unanswered. To start, we assume that the elements of $\mathcal{M}_{i}$ are distinct elements. This means that for each reconstruction, one subcarrier not chosen previously is reconstructed. To select appropriate transform coefficients and $\mathcal{M}_{i}$ jointly, we require a sensible optimization criteria that optimizes the performance in some sense and also admits a closed-form solution as well. We attempt to provide some possible answers in this section.

\footnotetext{
${ }^{1}$ When multiplied with a vector, the matrix $\mathcal{E}_{m}$ only excludes the $m$ th element of the vector, while the matrix $\boldsymbol{I}_{m}$ only includes the $m$ th element.
}

We make two simplifications to achieve our goal. We make the error free assumption (EFA), where we assume that in iteration $i$, the decisions made in previous iterations are correct, i.e., $\mathbf{e}_{m-1}=0, \forall m=1, \cdots, i-1$. We also restrict the class of transform to unitary transforms, which is appealing from the implementation and analysis point of view. Thus, we have the following.

$$
\text { Rule 1: } \boldsymbol{\Psi}=\mathbf{W}^{H} \Rightarrow \mathbf{W}^{H} \mathbf{W}=\mathbf{I} \text {. }
$$

This definition also ensures that the modulation symbol power $\sigma_{s}^{2}$ is properly normalized to the information symbol power $\sigma_{x}^{2}$, assuming that $E\left[\mathbf{x x}^{H}\right]=\sigma_{x}^{2} \mathbf{I}$ since

$$
E\left[\mathbf{s s}^{H}\right]=E\left[\mathbf{W} \mathbf{x x}{ }^{H} \mathbf{W}^{H}\right]=\sigma_{x}^{2} \mathbf{I} .
$$

Note that $v_{i, j}=0$ for $i=0$, as there is no error propagation for the initial initialization. The EFA implies that $v_{i, j}=0$ for iteration $i \geq 1$. From (12), this allows us to obtain the SNR as

$$
\gamma_{i j}=\frac{\bar{\gamma}}{\sum_{m \in \mathcal{M}_{i}^{C}} \frac{\left|w_{m j}\right|^{2}}{\left|h_{m}\right|^{2}}}
$$

where we have applied Rule 1 . We denote $\bar{\gamma}=\sigma_{x}^{2} / \sigma_{n}^{2}$ to be the average SNR.

The BER would be limited by the worse-performing symbol, especially at high SNR. Thus, we design the transform based on maximizing the minimum SNR $\gamma_{i j}$ for $j=1,2, \cdots, M$. This is subject to the condition that the actual values of a given set of channel amplitudes $\left\{\left|h_{m}\right|^{2}\right\}_{m=1}^{M}$ are fixed but not known at the transmitter. Thus, the solution is robust in the sense that it does not depend on the channel statistics; hence, it should work well in wireless channels with or without antenna diversity. Besides, since earlier iterations would lead to error propagation, the optimization should be carried out first for $i=0$, then sequentially for increasing $i$. Mathematically, the transform $\mathbf{W}$ and reconstruction indices $\mathcal{M}_{i}$ are selected as

$$
\arg \max _{\mathbf{W}, \mathcal{M}_{i}} \min _{j} \gamma_{i j}=\arg \min _{\mathbf{W}, \mathcal{M}_{i}} \max _{j} \sum_{m \in \mathcal{M}_{i}} \frac{\left|w_{m j}\right|^{2}}{\left|h_{m}\right|^{2}}
$$

subject to Rule 1 (which constraints the transmission power) for $i=0,1,2, \cdots$ in a sequential manner.

Consider the case when $i=0$. In this case, the optimization is carried out for $\mathbf{W}$ only since $\gamma_{0 j}$ is not a function of $\mathcal{M}_{i}$. For a given set of channel amplitude $\left\{\left|h_{m}\right|^{2}\right\}_{m=1}^{M}$ whose order is not known a priori at the transmitter, to satisfy (14), the minimax solution is given by $\left|w_{m j}\right|^{2}=k^{2}$, a positive constant for $j, m=$ $1,2, \cdots M$. Thus, we may restate this requirement as follows.

Rule 2: $w_{n m}=(1 / \sqrt{M}) e^{j \theta_{n m}}, n, m=1,2, \cdots, M$.

The set of arbitrary angles $\left\{\theta_{n m}\right\}$ is constrained to satisfy Rule 1.2 Using an LS detector, this transform design based on maximizing the minimum SNR results in the same transform design as given in [16], when the BER is minimized for high SNR. However, our results are applicable for any channel, while [16] considers only a Rayleigh-fading channel. Note that the design is significantly different when a different detection method is employed, such as for the case when the ML detector is used, as illustrated in [17]. In general, our transform may not lead to optimum performance when a different detector is used, and sim-

\footnotetext{
${ }^{2}$ This rule is violated by the OFDM system, where the PT is equal to the identity matrix.
} 
ilarly, a transform designed for ML detection may not be optimum for our purpose here.

Thus, the SNR is uniform in the sense that it attains the same value for all $j$ for $i=0$. In fact, this is true for subsequent iterations as well. In general, the SNR (as optimized for $i=0$ ) can be written independent of $j$ as

$$
\gamma_{i j}=\frac{M \bar{\gamma}}{\sum_{m \in \mathcal{M}_{i}^{C}}\left|h_{m}\right|^{-2}} .
$$

For iteration $i \geq 1$, we determine the reconstruction indices $m_{i}$ using the same optimization criteria (14) substituted with (15); thus, we are required simply to maximize $\gamma_{i j}$. We define $g_{m} \in\left\{h_{l}, l=1, \cdots M\right\}, m=1, \cdots M$ such that each $g_{m}$ corresponds uniquely to one $h_{m}$ and $\left|g_{1}\right|^{2} \leq\left|g_{2}\right|^{2} \leq \cdots \leq\left|g_{M}\right|^{2}$. Therefore, $\left\{g_{m}\right\}$ is simply $\left\{h_{m}\right\}$ but reordered such that the first index corresponds to the channel that gives the smallest amplitude, the second index gives the second-smallest amplitude, and so on. It is easy to see that the SNR in (15) is largest when $\mathcal{M}_{i}$ is chosen such that $m_{i} \in \mathcal{M}_{i}$ corresponds to the smallest $\left|h_{m_{i}}\right|^{2}$. Therefore

$$
\max \gamma_{i j}=\frac{M \bar{\gamma}}{\sum_{m=i+1}^{M}\left|g_{m}\right|^{-2}} \triangleq \gamma_{i}, \quad i=0,1,2, \cdots
$$

which is still independent of $j$. In order to maximize the SNR after the initial iteration, we conclude that for iteration $i$, we should always reconstruct the weakest subcarrier that has not been previously chosen.

\section{Flexibility in Transform Design}

The given transform design has some flexibility. A total of $M^{2}$ angles $\left\{\theta_{n m}\right\}$ can be chosen to satisfy Rule 2 , subject to the constraint of Rule 1 . The choice can be made based on considerations to minimize implementation complexity, reduce peak-to-average power ratio (PAPR), or simply to harmonize with current standards. We describe two well-known transforms here.

1) Walsh Hadamard Transform (WHT): If the PT matrix equals the WHT matrix (known as WHT-OFDM), the columns are orthogonal Walsh Hadamard spreading codes. It is well know that the WHT matrix of order $M$ can be constructed by an iterative procedure

$$
\mathbf{W}_{2}=\frac{1}{\sqrt{2}}\left[\begin{array}{cc}
1 & 1 \\
1 & -1
\end{array}\right] ; \quad \mathbf{W}_{2^{k}}=\mathbf{W}_{2^{k-1}} \otimes \mathbf{W}_{2}
$$

where $\otimes$ denotes the Kronecker product. A WHT-OFDM becomes an MC-CDMA system when the spreading code size is the same as the number of subcarriers, $M$.

2) FFT: If we set $\mathbf{W}$ to be the FFT matrix ${ }^{3}$ instead, then we obtain an SC-FDE system, which is noted in [16] as well. In this case, the PAPR would be very small, depending on the PAPR of the original information symbols.

\section{E. Algorithm Refinement}

The algorithm is designed using a linear LS filter, followed by a hard decision function and one reconstruction for each iteration. The performance can be improved by using the following

\footnotetext{
${ }^{3}$ We assume that the FFT is normalized to fulfill Rule 1.
}

refinements in the actual implementation, as verified in the simulations conducted in Section VI.

1) Use of Linear MMSE Filter: Instead of the LS filter, the MMSE filter can be used, which is designed to minimize the MSE of the error vector after filtering. Under the EFA, the solution for the filter is a function of $i$ and given as

$$
\mathbf{G}_{i}=\mathbf{W}^{-1} \mathbf{B}_{i}
$$

where $\mathbf{B}_{i}$ is a diagonal matrix defined in Appendix A. Appendix A shows that the transform design and reconstruction criteria derived for the LS filter is still valid when the MMSE filter is used. It is to be noted that for weak subcarriers with small $\left|h_{m}\right|,\left|b_{m}\right|$ is small to avoid the excessive noise amplification. However, for strong subcarriers with large $\left|h_{m}\right|, b_{m}$ becomes closer to $1 / h_{m}$, which reduces interference due to loss of orthogonality. Therefore, the MMSE detector compromises between effects of excessive noise amplification and interference due to loss of orthogonality and, thus, outperforms the LS detector in most communication systems.

2) Use of Soft Decision Function: The decision function at each iteration can be altered to improve the performance. For example, the clipping function for BPSK signals may be given as

$$
\widehat{x}=\left\{\begin{array}{cc}
\widetilde{x}, & |\widetilde{x}|<x_{R} \\
\operatorname{sign}(\widetilde{x}), & |\widetilde{x}| \geq x_{R}
\end{array}\right.
$$

where $\operatorname{sign}(\cdot)$ is the sign function taking value $\pm x_{R}$, and $x_{R}$ denotes the real part of the transmitted signal. For quadrature phase shift keying (QPSK) signals, the clipping function in (19) may be applied to real and imaginary part separately. By using the clipping function, hard decision is made only for those bits with more energy that are relatively robust to noise. For bits with less energy and, thus, more susceptible to an erroneous decision, decisions are not made with the hope that further iterations would assist in improving decisions made in the last iteration.

3) Iterate Using the Same Reconstruction Subcarrier: The iterative process can also be applied to one particular subcarrier a few times for the effects of the reconstruction to be fully realized before the next subcarrier is reconstructed. However, based on simulation results, it will not get much improvement usually after three or four repetitions.

\section{Performance Analysis Under EFA}

In this section, we consider the derivation of the upper and lower BER bounds under EFA. The lower BER bound under the EFA is the true lower bound of the actual BER. The derivation of the upper bound under EFA is a by-product of the analysis. However, with knowledge of the upper bound, we can determine the diversity of the iterative detector under EFA. Practically, the EFA is never valid, but it allows us to understand the behavior of the iterative detector under optimistic conditions. Also, it is expected that the EFA can be well approximated when channel codes are introduced and the output of the channel decoder fed back to the detector.

We obtain the BER bounds by first bounding the SNR appropriately using an LS filter. The objective is to obtain a closedform expression that produces insights on the performance of 
the system. Similar derivations for the BER can be applied using MMSE filter; however, since only the signal-to-(interference plus noise) ratio (SINR) can be calculated directly, where the interference is non-Gaussian, the BER obtained via the SINR serves only as an approximation.

The SNR $\gamma_{i}$ defined in (16) is bounded for the $i$ th iteration as

$$
\gamma_{\mathrm{low}, i} \leq \gamma_{i} \leq \gamma_{\mathrm{up}, i}
$$

where

$$
\gamma_{\mathrm{up}, i} \triangleq M \bar{\gamma}\left|g_{i+1}\right|^{2}, \quad \gamma_{\mathrm{low}, i} \triangleq \frac{M \bar{\gamma}\left|g_{i+1}\right|^{2}}{(M-i)}
$$

This bound can be easily obtained by noting that $\left|g_{i+1}\right|^{-2} \geq$ $\left|g_{i+2}\right|^{-2} \geq \cdots \geq\left|g_{M}\right|^{-2}$. Since $\gamma_{\text {low }, i}$ is simply $\gamma_{\text {up }, i}$ scaled by $(M-i)$ times, the BER based on $\gamma_{\text {low }, i}$ can be obtained easily once the BER based on $\gamma_{\mathrm{up}, i}$ is known. In addition, the SNR difference between the bound is $10 \log _{\{10\}} 10(M-1) \mathrm{dB}$, which improves as $M$ gets smaller or as $i$ gets larger.

\section{A. Channel Statistics}

For a single antenna system where no receive diversity can be capitalized, we assume that $h_{1}, h_{2}, \ldots, h_{M}$ are identical independent Gaussian distributed. The independence assumption is valid if we select adjacent subcarriers that are spaced more than the coherence bandwidth in frequency to form a group, and in this case, the model presented in (1) and (2) is used for the individual groups. For an $L \geq 2$ receive antenna system, we treat $\Gamma$ as the equivalent channel after maximal ratio combining (MRC) is applied. Thus, in general, $\left|h_{1}\right|^{2},\left|h_{2}\right|^{2}, \ldots,\left|h_{M}\right|^{2}$ can be modeled as identical independent central chi-square distributed random variable with $2 L$ degrees of freedom, where $E\left[\left|h_{m}\right|^{2}\right]=L, \forall m$. For the case when channel diversity is provided by the multiple transmit antennas through orthogonal space-time block codes [27], the analysis below can be applied similarly since the channel statistics are the same, except for a simple variable change to account for differences in the mean of the SNR.

The SNR of a subcarrier is denoted as $\gamma=\alpha|h|^{2}$. The constant $\alpha>0$ equals the average SNR $\bar{\gamma}$ for a conventional OFDM system. With the above considerations, the probability density function (PDF) of $\gamma$ is, therefore, given as [28]

$$
f_{\gamma}(\gamma)=\frac{\gamma^{L-1}}{(L-1) ! \alpha^{L}} \exp \left(-\frac{\gamma}{\alpha}\right)
$$

The cumulative density function (cdf) $F_{\gamma}(\gamma)$ can be obtained by performing integration by parts on (21) $L-1$ times

$$
F_{\gamma}(\gamma)=1-\sum_{l=0}^{L-1}\left(\frac{\gamma}{\alpha}\right)^{l} \exp \left(-\frac{\gamma}{\alpha}\right)
$$

\section{B. Conventional OFDM System}

We first consider the BER of an OFDM system with an $L$ th-order diversity channel to be used for comparison with a PT-OFDM system. Let the BER of a modulated system in an
AWGN channel be $P_{\text {awgn }}(\gamma)$. Using the PDF of the SNR given in (21) with $\alpha=\bar{\gamma}$, the BER of an Lth-order diversity channel is

$$
P_{L}^{\text {fading }}(\bar{\gamma})=\int_{0}^{\infty} P_{\text {awgn }}(\gamma) f_{\gamma}(\gamma \mid \alpha=\bar{\gamma}) d \gamma
$$

For common modulation schemes such as MQAM and MPSK schemes, closed-form expressions or expressions amenable to numerical calculation are available [6], [28]. We consider the specific case of a QPSK modulated system, where the closedform expression of the BER is

$$
P_{L}^{\text {fading }}(\bar{\gamma})=\left(\frac{1-\mu}{2}\right)^{L} \sum_{l=0}^{L-1}\left(\begin{array}{c}
L-1+l \\
l
\end{array}\right)\left(\frac{1+\mu}{2}\right)^{l}
$$

where $\mu=\sqrt{\bar{\gamma} /(1+\bar{\gamma})}$

\section{PT-OFDM System}

In order to bound the BER, we need to obtain the PDF of $\gamma_{\text {low }, i}$ and $\gamma_{\text {up }, i}$. This is done by first considering in general for some constant $\alpha$, the distribution of the ordered SNR $\tilde{\gamma}_{i}=$ $\alpha\left|g_{i}\right|^{2}$, denoted as $f_{\tilde{\gamma}_{i}}(\gamma)$. The results are given in Appendix B as (38) and (39) for the case of $L=1,2$, respectively. The extensions of the results for $L \geq 3$ can be carried out similarly. With the substitutions $\bar{\gamma}_{\text {low }, i} \triangleq M \bar{\gamma} /(M-i)$ and $\bar{\gamma}_{\text {up }, i} \triangleq M \bar{\gamma}$, the PDF of the lower and upper SNR bounds defined in (20) can then be easily obtained, respectively, as

$$
\begin{aligned}
f_{\gamma_{\text {low }, i}}(\gamma) & =f_{\tilde{\gamma}_{i}}\left(\gamma \mid \alpha=\bar{\gamma}_{\text {low }, i}\right) \\
f_{\gamma_{\text {up }, i}}(\gamma) & =f_{\tilde{\gamma}_{i}}\left(\gamma \mid \alpha=\bar{\gamma}_{\text {up }, i}\right) .
\end{aligned}
$$

Now, we are ready to consider the BER for the iterative PT-OFDM system. Using the SNR $\gamma_{i}$ defined in (16), the exact BER for the $i$ th iteration is given by a multifold integration

$$
P_{\mathrm{e}}(i, \bar{\gamma})=\int_{\mathbf{g}_{i}} P_{\text {awgn }}\left(\gamma_{i} \mid \mathbf{g}_{i}\right) f_{\mathbf{g}_{i}}\left(\mathbf{g}_{i}\right) d \mathbf{g}_{i}, \quad i=0,1, \cdots M-1
$$

where we define $\mathbf{g}_{i}=\left[\left|g_{i+1}\right|^{2},\left|g_{i+2}\right|^{2}, \cdots,\left|g_{M}\right|^{2}\right]^{T}$, and $f_{\mathbf{g}_{i}}(\cdot)$ is its multivariate PDF. A closed-form expression cannot be obtained easily for the general case, even for the case when $i=0$. However, since the instantaneous SNR is lower (upper) bounded by $\gamma_{\text {low }, i}\left(\gamma_{\text {up }, i}\right)$ given in (20), the instantaneous BER can be upper (lower) bounded. Taking the expectation of the instantaneous BER, we are then able to bound the average BER in an $L$ th-order diversity channel. We first consider the derivation of the upper bound BER as follows:

$$
P_{\mathrm{e}}(i, \bar{\gamma}) \leq P_{\mathrm{up}}(i, \bar{\gamma}) \triangleq \int_{0}^{\infty} P_{\mathrm{awgn}}(\gamma) f_{\gamma_{\mathrm{low}, i}}(\gamma) d \gamma
$$

where only a single-fold integration needs to be carried out. Substituting (25) into (38) and (39), a closed-form expression can be found as shown next for $L=1$ and 2 for QPSK modulated symbols. 
Consider $L=1$. Using (23), (28), and (38), we obtain after some manipulations the upper bound BER

$P_{\text {up }}(i, \bar{\gamma})=\frac{M !}{i !(M-i-1) !} \sum_{k=0}^{i}\left(\begin{array}{l}i \\ k\end{array}\right) \frac{(-1)^{k}}{\beta} P_{1}^{\text {fading }}\left(\frac{\bar{\gamma}_{\text {low }, i}}{\beta}\right)$

where $\beta=M-i+k$. This explicit formulation of the BER in terms of $P_{L}^{\text {fading }}$ where $L=1$ is advantageous since we can make use of the rich literature investigating (23) for different scenarios. In this case, closed-form expressions exist for many common modulation schemes, such as for QPSK as given in (24), and thus, the upper bound BER has a closed-form expression for such cases as well. The lower bound BER, denoted as $P_{\text {low }}(i, \bar{\gamma})$, can be derived similarly by using $\gamma_{\text {up }, i}$. Alternatively, note that since the upper and lower bound SNR differs by a factor of $M-i$, we can immediately derive it as

$$
P_{\text {low }}(i, \bar{\gamma})=P_{\text {up }}(i,(M-i) \bar{\gamma}) \text {. }
$$

Next, consider $L=2$. After similar manipulations, we can also obtain the upper bound BER for iteration $i$

$$
\begin{aligned}
& P_{\text {up }}(i, \bar{\gamma})=\frac{M !}{i !(M-i-1) !} \sum_{k=0}^{i}\left(\begin{array}{c}
i \\
k
\end{array}\right) \sum_{n=0}^{\beta-1}\left(\begin{array}{c}
\beta-1 \\
n
\end{array}\right) \\
& \times \frac{(-1)^{k}(n+1) !}{\beta^{n+2}} P_{n+2}^{\text {fading }}\left(\frac{\bar{\gamma}_{\text {low }, i}}{\beta}\right) \text {. }
\end{aligned}
$$

This is expressed in terms of $P_{n+2}^{\text {fading }}$ given by (23) for $L=$ $n+2$. The lower bound BER is

$$
P_{\text {low }}(i, \bar{\gamma})=P_{\text {up }}(i,(M-i) \bar{\gamma}) .
$$

For $L>2$, similar derivations can be carried out with an increase in the number of summation operators.

We formally summarize the main results in the following theorem.

Theorem 1: Let the equivalent channel PDF be given by (21). With the error-free assumption for $i \geq 1$ and, in general for $i=0$, the exact BER for the $i$ th iteration can be bounded as

$$
P_{\text {low }}(i, \gamma) \leq P_{e}(i, \gamma) \leq P_{\text {up }}(i, \gamma)
$$

where $P_{\text {up }}(i, \gamma)$ and $P_{\text {low }}(i, \gamma)$ are given by (29) and (30), respectively, for $L=1$ and given by (31) and (32), respectively, for $L=2$.

\section{Performance Comparison With Conventional Scheme}

In this section, we make some analytical comparisons with the conventional scheme. In some cases when simple comparison is not possible, asymptotic results are presented.

In order for us to derive some useful results, we assume that the BER of the $L$ th-order diversity channel given by (24) can be expressed as

$$
P_{L}^{\text {fading }}(\bar{\gamma})=\sum_{j=L}^{\infty} \frac{a_{j}}{\bar{\gamma}^{j}}
$$

for some constants $a_{j}, j=L, L+1, \cdots \infty$. This is shown to be possible for a QPSK modulated system in Appendix C for $L=1,2$. In general, it is also easy to show that (33) is valid for MQAM and QPSK modulated systems for any integer $L$.

The results below hold true when the transform satisfies Rules 1 and 2 and when LS detection is employed at the receiver. The EFA is used when reconstruction are carried out, i.e., when $i>$ 0 . All proofs for the corollaries are given in Appendix D.

1) Initial Iteration, $i=0$ : For the case when $i=0$, Theorem 1 is exact in the sense that it does not rely on the EFA. When $L=1$, we should always use a conventional OFDM system instead of a PT-OFDM system, since the conventional OFDM system performs at least as good as the PT-OFDM system in terms of BER, as shown by Corollary 1 . This result holds true for a variety of systems, such as MC-CDMA or SC-FDE systems, where no iteration is carried out and is, thus, useful in its own right.

Corollary 1: Define $P_{\mathrm{e}}(0, \bar{\gamma})$ as the exact BER of the PT-OFDM system when no iteration is performed and $P_{1}^{\text {fading }}(\bar{\gamma})$ as the BER of the conventional OFDM system in a single antenna system. Then, $P_{\mathrm{e}}(0, \bar{\gamma}) \geq P_{1}^{\mathrm{fading}}(\bar{\gamma})$.

For a system with $L$ th channel diversity, where $L \geq 2$, Corollary 1 does not hold in general. Instead, motivated by the simulation results in Section VI that the exact BER approaches the lower bound BER at high SNR for $L=2$, we present the following corollary to explore the asymptotic performance.

Corollary 2: $P_{\text {low }}(0, \bar{\gamma}) \rightarrow(1 / M) P_{2}^{\text {fading }}(\bar{\gamma})$ as SNR $\rightarrow$ $\infty$, assuming that (33) is valid.

For $i=0, L=2$, the asymptotic lower bound BER of the PT-OFDM system is, therefore, $M$ times smaller as compared to the BER of a conventional OFDM system. With Corollary 2, we conclude that the use of a PT is able to exploit the channel diversity to achieve better performance when $M$ is large and SNR is sufficiently high.

2) Diversity Advantage of Iterative Detection in PT-OFDM System: For the case when $L=1$, Corollary 1 suggests that additional measures have to be taken to improve the performance of the PT-OFDM system. By using the EFA, we provide the theoretical motivation for the advantage of the proposed iterative method by Corollary 3 .

Corollary 3: For $L=1$, the iterative detector described provides a diversity advantage of $i+1$ after completing $i$ iteration, assuming perfect reconstruction. The diversity advantage is defined as $\lim _{\bar{\gamma} \rightarrow \infty}(-\log (\mathrm{BER}) / \log (\bar{\gamma}))$, which measures the asymptotical rate of decrease of the BER as the average SNR $\bar{\gamma}$ increases.

\section{OTHER ISSUES}

\section{A. Complexity}

We next consider the complexity of the algorithm, measured as the number of required complex multiplications and divisions. The complexity of the detection stage is not considered since it is relatively simple to implement. In addition, we do 
TABLE I

COMPleXity of the Proposed Iterative Detector IN TeRMS OF Multiplications $(\times)$ AND Divisions $(\div)$

\begin{tabular}{c|c|c}
\hline Iteration $i$ & Iterative LS detector & Iterative MMSE detector \\
\hline 0 & $M^{2}+M(\times), M(\div)$ & $M^{2}+2 M(\times), M(\div)$ \\
\hline$\geq 1$ & $2 M+2(\times)$ & $3 M+2(\times), 1(\div)$ \\
\hline \hline Total for $I$ iterations & $\leq M^{2}+M+I(2 M+2)(\times), \leq M(\div)$ & $\leq M^{2}+2 M+I(3 M+2)(\times), \leq M+I(\div)$ \\
\hline
\end{tabular}

not make any assumption on the elements of $\mathbf{W}$ and $\hat{\mathbf{x}}_{i}$. Further complexity reduction can be made, for example, when the symbols are QPSK modulated or when a well-structured matrix such as the FFT matrix is used.

Consider $i=0$. The reconstruction stage is not required. The computation of $\boldsymbol{\Gamma}^{-1}$ requires $M$ divisions, and $\mathbf{B}_{0}$ requires $M$ divisions and $M$ multiplications. For LS filtering, obtaining $\widetilde{\mathbf{x}}_{0}$ requires $M+M^{2}$ multiplications if carried out from right to left as $\mathbf{W}^{H} \boldsymbol{\Gamma}^{-1} \mathbf{r}$. The same number of multiplications is required for MMSE filtering. The result is tabulated in Table I.

For $i \geq 1$, the complexity of the algorithm, as summarized in Section III-B, can be further simplified. First, we note from Appendix A that $\mathbf{B}_{i}=\mathcal{E}_{m_{i}} \mathbf{B}_{i-1}+\frac{1}{h_{m_{i}}} \boldsymbol{I}_{m_{i}}$ for $i \geq 1$. By using the fact that $\mathcal{E}_{m}=\mathbf{I}-\mathcal{I}_{m}$, after some algebraic manipulations, the algorithm can be implemented recursively as

- reconstruction: $\mathbf{r}_{i}=\mathbf{r}_{i-1}-\mathbf{c}_{i}$

- filtering: $\widetilde{\mathbf{x}}_{i}=\widetilde{\mathbf{x}}_{i-1}-\mathbf{G}_{i} \mathbf{c}_{i}+\mathbf{d}_{i}$

- detection: $\hat{\mathbf{x}}_{i}=\operatorname{dec}\left(\widetilde{\mathbf{x}}_{i}\right)$

where

$$
\mathbf{c}_{i}=\mathcal{I}_{m_{i}}\left(\mathbf{r}_{i-1}-\Gamma \mathbf{W} \hat{\mathbf{x}}_{i-1}\right) .
$$

For LS filtering

$$
\mathbf{G}_{i}=\mathbf{W}^{H} \boldsymbol{\Gamma}^{-1}, \mathbf{d}_{i}=\mathbf{0}
$$

while for MMSE filtering

$$
\mathbf{G}_{i}=\mathbf{W}^{H} \mathbf{B}_{i}, \mathbf{d}_{i}=\mathbf{W}^{H}\left(\frac{1}{h_{m_{i}}}-\frac{h_{m_{i-1}}}{\left|h_{m_{i-1}}\right|^{2}+\sigma_{n}^{2}}\right) \mathcal{I}_{m_{i}} \mathbf{r}_{i-1} .
$$

The required number of operations can be calculated similarly for $i \geq 1$. By reusing previous calculated results and performing only necessary operations (especially when $\boldsymbol{I}_{m}$ and diagonal matrices are involved), the complexity is obtained as shown in Table I. We omit the lengthy but straightforward calculations to obtain the numbers. Some minor differences are expected for different implementation; however, the order of the complexity would not be affected.

The complexity for a total of $I$ iterations (where $I$ is the number of reconstructions multiplied by the number of repetitions for each reconstruction) is shown in Table I. We ignore the possibility of reducing some computations when repetitions are performed. We observe that for $i=0$, the complexity is quadratic, while for $i \geq 1$, the complexity is linear. Also, when $I$ is much larger than $M$, we observe that the overall complexity is approximately linear. When $I$ is of the same order as $M$, which is usually the case for the PT-OFDM system, then the complexity is approximately quadratic. From Table I, we see that each iteration only adds a marginal complexity.

\section{B. Channel Not Known Perfectly}

In practical implementations, the channel is not known exactly at the receiver but needs to be estimated via a training sequence. The iterative detection schemes are not particularly sensitive to channel estimation errors. When an LS channel estimator is used for simulations, the same amount of degradation is experienced by conventional OFDM and PT-OFDM systems with or without iterations. Thus, the same performance superiority is maintained over conventional systems.

\section{Coded Performance}

The iterative detector can be concatenated with a channel code to further improve its performance. Depending on the code used, it can lead to a varying degree of coding gain. The iterative detector can be further optimized with respect to the channel codes as well.

\section{Simulation Results}

\section{A. Performance Under EFA and Independent Subcarriers}

In order to verify and reinforce the conclusions made from the analysis in Section IV, we perform simulations for the PT-OFDM system under the EFA. The EFA is not required when $i=0$, as noted in Section IV. The number of time-domain channel taps is equal to the size of the matrix, and we assume a uniform power delay profile (i.e., i.i.d. channels for the subcarriers). We use WHT for the transform.

For $L=1$, as a test case, we set $M=8$ and $i=0$, $1,2,3$. We simulate the performance of the QPSK modulated WHT-OFDM system. The results are given in Fig. 2. It is seen that the simulated BERs fall between their respective upper and lower bounds, as predicted in Theorem 1. It is also verified from simulations that the conventional OFDM system has the same BER as the lower bound of PT-OFDM for $i=0$ (not shown in the figure for the sake of clarity), thus validating Corollary 1. Last, it appears that the BERs converge to the lower bound at high SNR, hence achieving a diversity advantage of $i+1$ at iteration $i$. This agrees with Corollary 3 . We emphasize that the performance is very optimistic and serves to highlight the mechanism and potential benefit of the algorithm.

For $L=2$, we use $M=2,4,16$, and $i=0$. The simulation results are shown in Fig. 3. It is seen that increasing $M$ would be sufficient to increase the performance of a PT-OFDM system when combined with diversity techniques. At high SNR $(>14$ $\mathrm{dB}$ ), it is seen that the performance would approach the one predicted by Corollary 2 . 


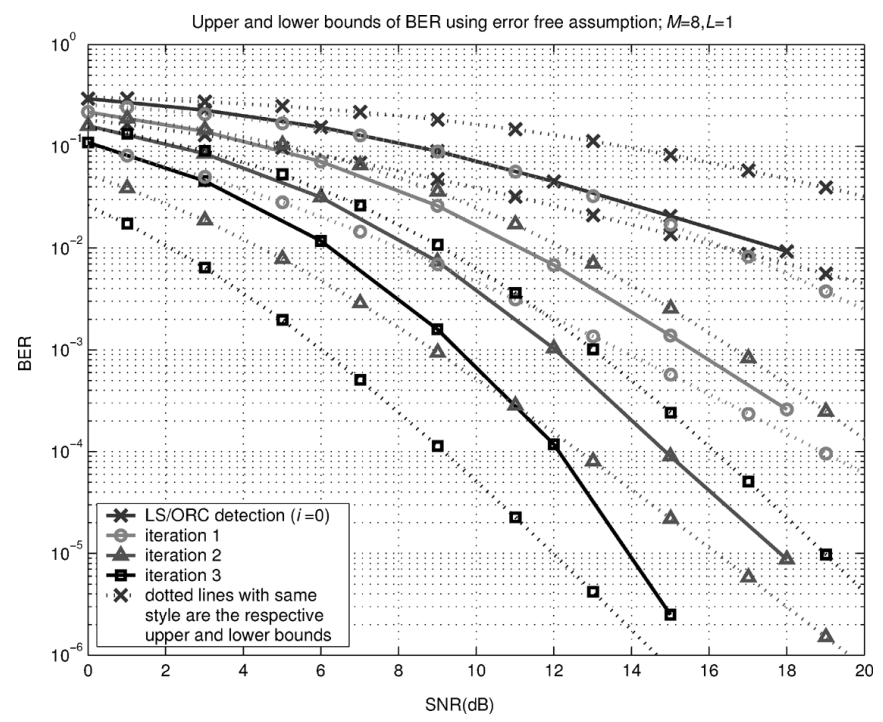

Fig. 2. Iterative detection BER bounds of WHT-OFDM system with $M=8$, $L=1$.

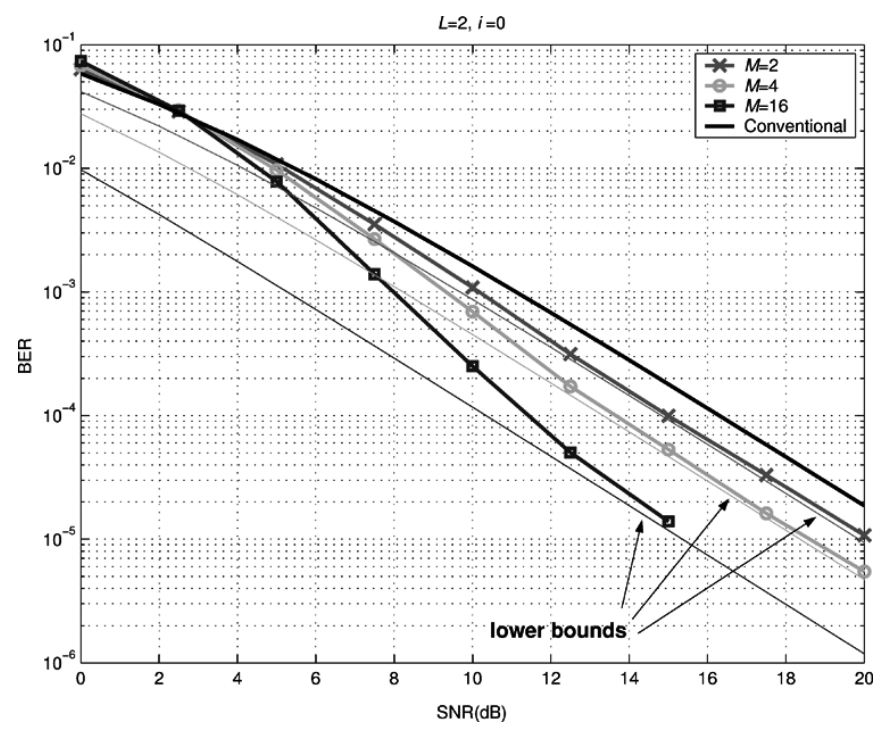

Fig. 3. BER lower bounds of WHT-OFDM system with $L=2$.

\section{B. Performance in Practical Scenarios}

In this section, we present simulation results of the PT-OFDM system in a practical frequency-selective fading channel. We assume that the duration of the cyclic prefix is the same as the maximum delay spread. The channel is modeled as an order $L_{h}=16$ FIR filter with exponential power delay profile and root mean-squared delay spread $\tau_{\text {rms }}=4$ (normalized to the sample interval). The number of subcarriers is set to $M=64$. For each iteration, four reconstructions are repeated on the same subcarrier to ensure convergence.

Fig. 4 illustrates the BER performance of the iterative LS detectors of a PT-OFDM system with hard decision versus SNR. The BER of a conventional LS detector for the OFDM systems is plotted as well. The iterative detector improves the BER performance significantly as more iterations are carried out. For BER at $10^{-2}$, the iterative algorithm using four worse subcarriers for reconstructions outperforms by 6 and $4 \mathrm{~dB}$, respectively, to the PT-OFDM and OFDM systems without

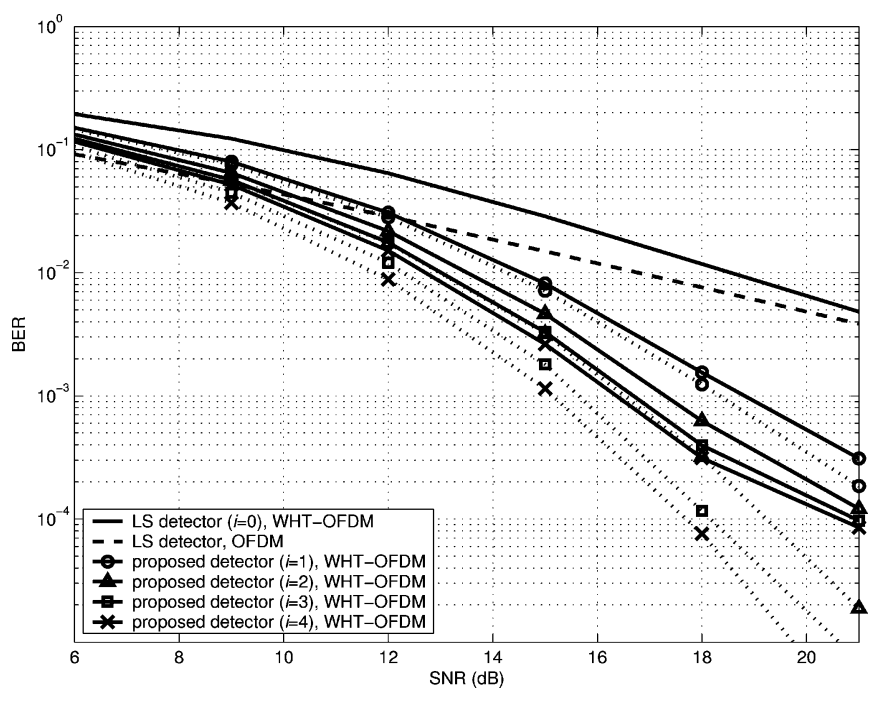

Fig. 4. Performance of the iterative LS detector with hard decision. The dotted lines show the simulated bounds under the EFA.

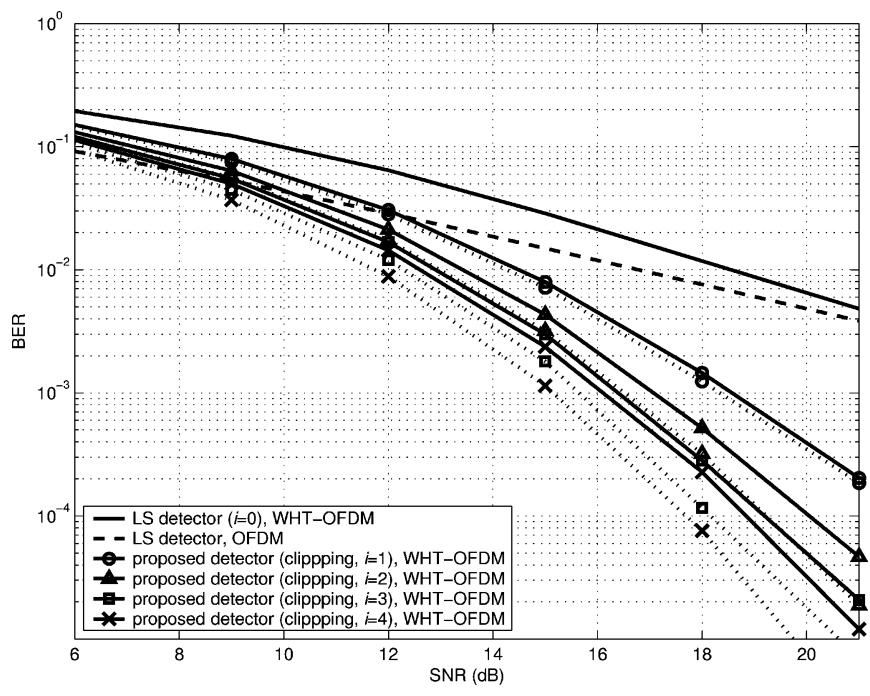

Fig. 5. Performance of the iterative LS detector with clipping function.

reconstruction. Even with only one reconstruction, it outperforms its counterparts by 4 and $2 \mathrm{~dB}$, respectively. As more subcarriers are reconstructed, the rate of improvement diminishes. Therefore, reconstructing a few worst subcarriers, which most influence overall performance, is sufficient to obtain desired performance improvement, and this makes the iterative detector easy to implement.

The dotted lines in Fig. 4 show the simulated bounds under the EFA. It shows that there is a gap between the actual performance and the bound achieved using EFA. This gap can be reduced partly by using a clipping function instead of a hard decision in each iteration. This is shown in Fig. 5. The improvement for the BER at $10^{-3}$ is trivial compared with using hard decision. At higher SNR area, the improvement is more significant. It can be seen that the performance of the proposed algorithm approximates the EFA bound well, especially for the first few iterations.

When the SNR is known at the receiver, significantly better performance can be obtained by using an MMSE filter, as illus- 


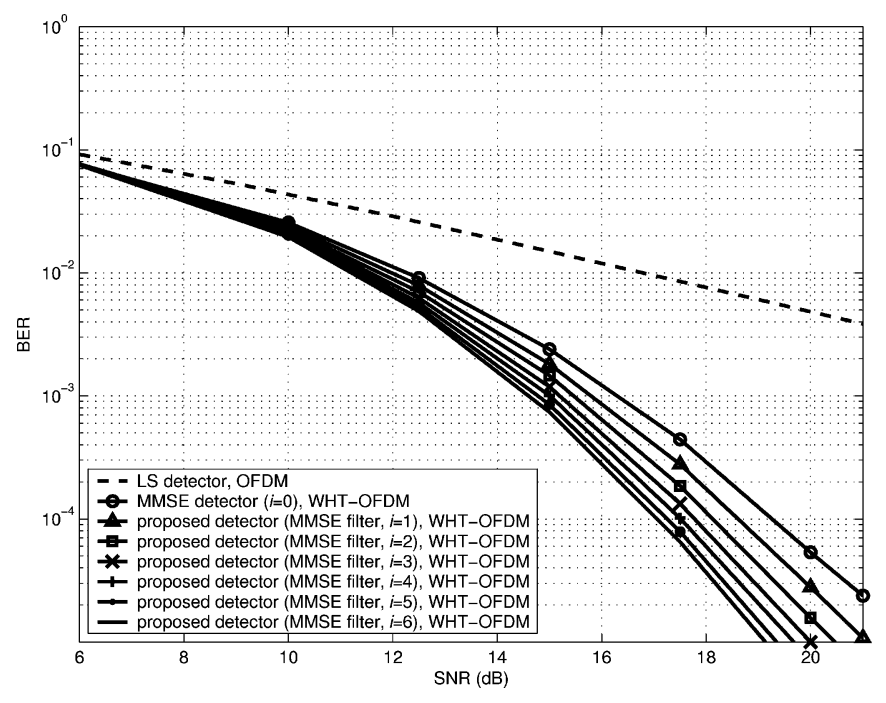

Fig. 6. Performance of the iterative MMSE detector with hard decision.

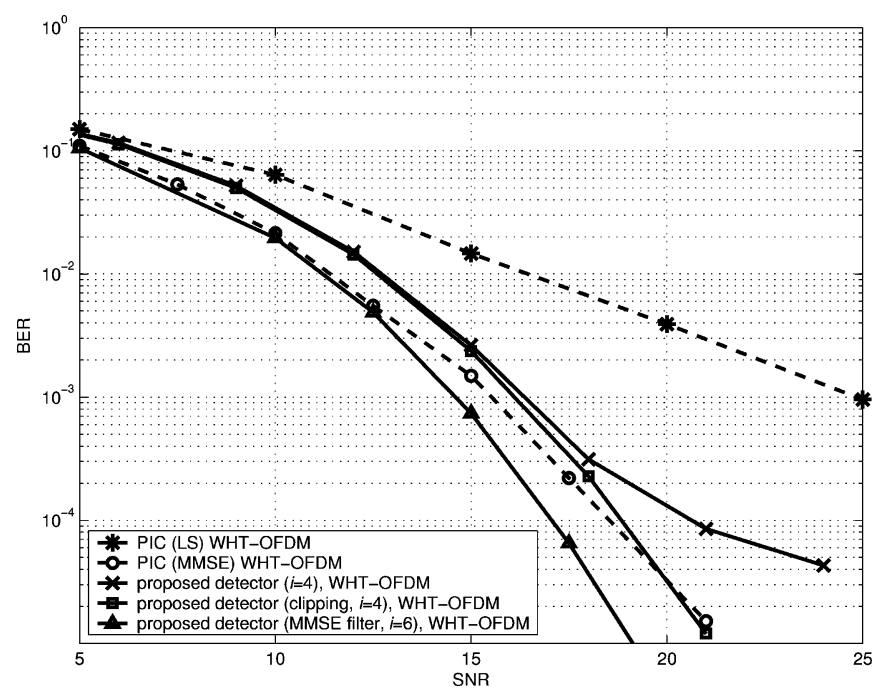

Fig. 7. Performance of the iterative detector compared with parallel interference cancellation scheme.

trated in Fig. 6. When $i=0$, the iterative MMSE detector is simply the classical MMSE filter. At BER of $10^{-4}$, approximately $2.5 \mathrm{~dB}$ of performance gain is achieved using six iterations over the classical MMSE detector. The performance gain is even more significant when compared to the LS detector for an OFDM system.

Next, we compare the performance shown in the previous figures with the PIC detector that is commonly used for multiuser detection [19], [20]. The initial stage of the PIC is the same as the initial iteration of the proposed iterative detector. The initialization can be carried out either by an LS or MMSE detector. Subsequently, the $i$ th stage of the PIC can be written as

$$
\widetilde{x}_{i, m}=\mathbf{h}_{m}^{H}\left(\mathbf{r}-\Gamma \mathbf{W} \mathcal{E}_{m} \widehat{\mathbf{x}}_{i-1}\right), \quad m=1,2, \cdots, M
$$

where $\mathbf{h}_{m}$ is the $m$ th column of the matrix $(\boldsymbol{\Gamma W})$. The decision of the $m$ th symbol at the $i$ th stage is carried out as $\widehat{x}_{i, m}=$ $\operatorname{dec}\left(\widetilde{x}_{i, m}\right)$, where a hard decision is applied. We observed in simulations that the performance diverges after the first iteration, so only the performance at the first iteration is shown in

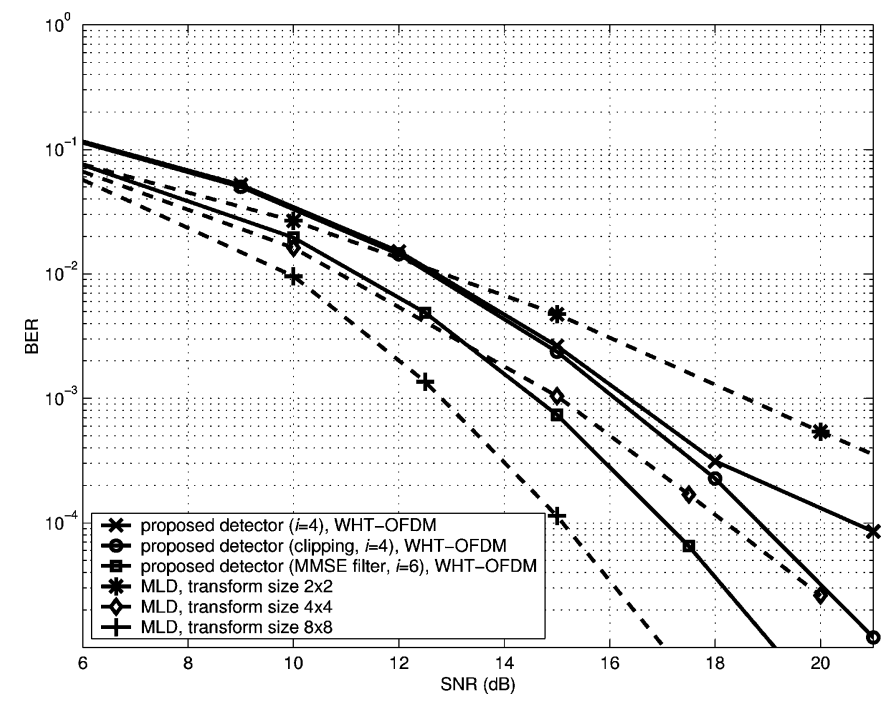

Fig. 8. Performance of the iterative detector compared with MLD detection.

Fig. 7. It is seen that the performance of the PIC is worse than the proposed iterative detector when both use either an LS or MMSE filter.

We consider the complexity of the PIC detector next. Calculating $\Gamma \mathbf{W} \mathcal{E}_{m} \widehat{\mathbf{x}}_{i-1}$ requires $M^{2}$ complex multiplications, and multiplying with $\mathbf{h}_{m}^{H}$ requires another $M$ multiplications. Thus, calculating $\widetilde{x}_{i, m}$ requires $M^{2}+M$ multiplications for one symbol. To detect all symbols, one stage of the PIC would then require $M\left(M^{2}+M\right)$ multiplications, which is of a cubic order of complexity. This is substantially more complex than the proposed iterative detector, since even when $I=6 \times 4$ total iterations are carried out, the complexity is still very low, as demonstrated in Section V.

Fig. 8 makes the comparison of the proposed iterative detectors with an ML detector designed in [17]. For ML detection, unitary square transform of size 2,4 , and 8 are used. The transformation is carried out over groups of subcarriers with the size matched to the transform used. The subcarriers in each group are selected such that the subcarrier indices are spaced as far apart as possible, as described in [17]. It is seen in the simulations that the performance for the ML detector depends on the size of the transform and could be better than using our proposed detector. However, the complexity of implementation is very high, in the order of $\mathcal{O}\left(4^{M}\right)$ when QPSK signals are used, and may be unacceptable. The order of complexity of the iterative detector, on the other hand, is linear to quadratic.

\section{CONCLUSION}

In this paper, a class of low-complexity iterative detectors is proposed for a PT-OFDM system. It works by reducing the overall system noise, especially due to noise amplification by trading weak subcarriers with estimated ones. We give the design rules for such a system by maximizing the minimum SNR of the symbols, giving rise to a family of transforms. We show that the use of additional techniques on top of the LS detection is necessary to improve the performance of a PT-OFDM system. We also prove that the proposed iterative detector leads to diversity advantage at high SNR under the assumption that 
the previous detections are correct. Finally, simulations in practical wireless channels demonstrate the superior performance of the iterative detector as compared to conventional detectors with comparable complexity.

\section{APPENDIX A}

\section{CONSIDERATIONS FOR THE MMSE FILTER}

In this Appendix, we consider the derivation of the MMSE filter and the SINR under the EFA. We then derive the transform design and reconstruction criteria by maximizing the minimum SINR. It is shown that by making the same simplifications as for the LS filter (i.e., Rule 1 and the EFA), we reach the same solution.

With no error propagation, we have $\hat{\mathbf{x}}_{i}=\mathbf{x}$, and from Section III-B, we can generalize the received vector at iteration $i$ as

$$
\mathbf{r}_{i}=\Gamma \mathbf{W} \mathbf{x}+\widetilde{\mathbf{n}}
$$

where the $m$ th element of $\widetilde{\mathbf{n}}$ is

$$
\widetilde{n}_{m}= \begin{cases}0, & m \in \mathcal{M} \\ n_{m}, & m \in \mathcal{M}^{C} .\end{cases}
$$

Without loss of generality, we assume that $\sigma_{x}^{2}=1$. Defining $\mathbf{H}=\Gamma \mathbf{W}$ and $\mathbf{R}_{\tilde{\mathbf{n}}}=E\left[\widetilde{\mathbf{n}} \widetilde{\mathbf{n}}^{H}\right]$, using standard derivations gives us the MMSE filter as $\mathbf{G}=\left[\mathbf{H}^{H} \mathbf{H}+\mathbf{R}_{\widetilde{\mathbf{n}}}^{\sim}\right]^{-1} \mathbf{H}^{H}$. Upon simplification, we obtain

$$
\mathbf{G}=\mathbf{W}^{H} \mathbf{B}_{i}
$$

where $\mathbf{B}_{i}=\operatorname{diag}\left(b_{1}, b_{2}, \cdots, b_{M}\right)$

$$
b_{m}= \begin{cases}h_{m}^{-1}, & m \in \mathcal{M}_{i} \\ \frac{h_{m}^{*}}{\left(\left|h_{m}\right|^{2}+\sigma_{n}^{2}\right)}, & m \in \mathcal{M}_{i}^{C}\end{cases}
$$

and $\sigma_{n}^{2}$ is the noise power.

Next, we consider the SINR of the signal after MMSE filtering $\widetilde{\mathbf{x}}_{i}=\mathbf{G r}_{i}$. After some algebraic manipulations, we obtain

$$
E\left[\widetilde{\mathbf{x}}_{i} \widetilde{\mathbf{x}}_{i}^{H}\right]=\mathbf{W}^{H} \mathbf{B}_{i} \boldsymbol{\Gamma} \mathbf{W}
$$

Thus, the sum of the signal, interference, and noise powers of the $j$ th symbol is given by the diagonal element of the matrix as $E\left[\left|\widetilde{x}_{i, j}\right|^{2}\right]=\sum_{m=1}^{M}\left|w_{j m}\right|^{2} h_{m} b_{m} \triangleq \delta_{j}>0$. Also, after filtering, the signal component of the $j$ th symbol can be extracted from $\widetilde{\mathbf{x}}_{i}=\mathbf{W}^{H} \mathbf{B}_{i}(\boldsymbol{\Gamma} \mathbf{W} \mathbf{x}+\widetilde{\mathbf{n}})$ by observation as $\mathbf{w}_{j}^{H} \mathbf{B}_{i} \Gamma \mathbf{w}_{j} x_{j}=\sum_{m=1}^{M}\left|w_{j m}\right|^{2} h_{m} b_{m} x_{j}$. The signal power is, therefore, $\delta_{j}^{2}$, and the SINR is given as

$$
\operatorname{SINR}=\frac{\delta_{j}^{2}}{\delta_{j}-\delta_{j}^{2}}=\frac{1}{\delta_{j}^{-1}-1}
$$

Following the same procedures used for an LS filter, we seek the solution for $\mathbf{W}$ and $\mathcal{M}_{i}$ by maximizing the minimum SINR over different $j$. By the same argument, we deduce that Rule 2 is required for $i=0$. This resulted in a uniform SINR across all $j$, regardless of the choice of $\mathcal{M}_{i}$. Then, we need to

$$
\begin{aligned}
\max \mathrm{SINR} & =\max \delta_{j} \\
& =\max \sum_{m \in \mathcal{M}_{i}^{C}} \frac{\left|h_{m}\right|^{2}}{\left|h_{m}\right|^{2}+\sigma_{n}^{2}}+\left|\mathcal{M}_{i}\right| \\
& =\max \sum_{m \in \mathcal{M}_{i}^{C}} \frac{1}{1+\frac{\sigma_{n}^{2}}{\left|h_{m}\right|^{2}}}
\end{aligned}
$$

where $\left|\mathcal{M}_{i}\right|$ is the cardinality of $\mathcal{M}_{i}$. Thus, for the SINR to be maximized, we need to choose $\mathcal{M}_{i}$ such that it corresponds to the set of indices corresponding to $i$ smallest channel amplitudes. This resulted in the same solution as for the case when the LS filter is used.

\section{APPENDIX B}

DERIVATION OF THE PDF OF $\tilde{\gamma}_{i}=\alpha\left|g_{i}\right|^{2}, \alpha>0$

This Appendix calculates the PDF of $\tilde{\gamma}_{i}=\alpha\left|g_{i}\right|^{2}$, formed by ordering $M$ independent realization of the random variable $\gamma=\alpha|h|^{2}$ from smallest to largest (i.e., $\gamma_{1} \leq \gamma_{2} \leq \cdots \leq \gamma_{M}$ ). Since the fading channels in all subcarriers are statistically independent, by using order statistics [29], the PDF of $\tilde{\gamma}_{i}$ is written as

$$
f_{\tilde{\gamma}_{i}}(\gamma)=\frac{M !}{i !(M-i-1) !} F_{\gamma}^{i}(\gamma)\left(1-F_{\gamma}(\gamma)\right)^{M-i-1} f_{\gamma}(\gamma)
$$

For the case of $L=1$, by substituting (21) and (22) into (37), we get

$$
\begin{aligned}
& f_{\tilde{\gamma}_{i}}(\gamma)=\frac{M !}{i !(M-i-1) ! \alpha} \sum_{k=0}^{i}\left(\begin{array}{l}
i \\
k
\end{array}\right)(-1)^{k} \\
& \times \exp \left(-\frac{\gamma(M-i+k)}{\alpha}\right)
\end{aligned}
$$

by performing the binomial expansion of $[1-\exp (-\gamma / \alpha)]^{i}$. For the case of $L=2$, after some manipulations, we get

$$
\begin{array}{r}
f_{\tilde{\gamma}_{i}}(\gamma)=\frac{M !}{i !(M-i-1) ! \alpha} \sum_{k=0}^{i}\left(\begin{array}{c}
i \\
k
\end{array}\right)(-1)^{k} \exp \left(-\frac{\gamma(M-i+k)}{\alpha}\right) \\
\times \sum_{n=0}^{M-i+k-1}\left(\begin{array}{c}
M-i-1+k \\
n
\end{array}\right)\left(\frac{\gamma}{\alpha}\right)^{n+1}
\end{array}
$$

where an additional binomial expansion is performed. Note that $f_{\tilde{\gamma}_{i}}(\gamma)$ are expressed in terms of the sums of different order terms of $e^{-\gamma}$ and $\gamma$ to facilitate latter derivations. In this similar manner, we can also derive the PDF for $L>2$.

\section{APPENDIX C \\ VALIDITY OF (33) FOR QPSK SIGNALS}

From (24), defining $x=1 / \bar{\gamma}$, we get $\mu=(1+x)^{-1 / 2}$. Clearly, the BER given by (24) is $m$ times differentiable at $x=$ $0, m=1,2 \cdots$, and thus, a Maclaurin series with coefficients denoted as $a_{m}$ corresponding to the $m$ th power can be formed as a power series of $x$. Note that for $m<L, a_{j}=0$ since substituting $x=0$ into $d / d x^{m}((1-\mu) / 2)^{L}$ as required in an 
intermediate step of the Maclaurin expansion gives a answer of 0 . Thus, we conclude that $P_{L}^{\text {fading }}(\bar{\gamma})=\sum_{j=L}^{\infty} a_{j} / \bar{\gamma}^{j}$.

In addition, from direct evaluation, the first few terms are given as $P_{L=1}^{\text {fading }}(\bar{\gamma})=1 /(4 \bar{\gamma})-3 /\left(16 \bar{\gamma}^{2}\right)+5 /\left(32 \bar{\gamma}^{3}\right)+\cdots$ and $P_{L=2}^{\text {fading }}(\bar{\gamma})=3 /(16 \bar{\gamma})-5 /\left(16 \bar{\gamma}^{2}\right)+105 /\left(256 \bar{\gamma}^{3}\right)-\cdots$.

\section{APPENDIX D \\ PROOF OF COROLLARIES}

\section{Proof of Corollary 1}

By definition, $P_{\mathrm{e}}(0, \bar{\gamma}) \geq P_{\text {low }}(0, \bar{\gamma})$. Substituting (29) into (30) for $i=0$, we get $P_{\text {low }}(0, \bar{\gamma})=P_{1}^{\text {fading }}(\bar{\gamma})$, thus concluding the proof that $P_{\mathrm{e}}(0, \bar{\gamma}) \geq P_{1}^{\text {fading }}(\bar{\gamma})$.

\section{Proof of Corollary 2}

From (31) and (32) and setting $i=0$, we get

$$
P_{\text {low }}(0, \bar{\gamma})=M \sum_{n=0}^{M-1}\left(\begin{array}{c}
M-1 \\
n
\end{array}\right) \frac{(n+1) !}{M^{n+2}} P_{n+2}^{\text {fading }}(\bar{\gamma}) .
$$

Using (33), for $L=2$, we see that $P_{\text {low }}(0, \bar{\gamma})$ can be asymptotically (as SNR $\rightarrow \infty$ ) represented by the first term of the summation of (40) at $n=0$. The proof is concluded since the first term is $1 / M$.

\section{Proof of Corollary 3}

To prove Corollary 3, we first need to prove the following Lemma.

Lemma 1: For any positive integer $N>n$

$\Lambda(j)=\sum_{k=0}^{n}\left(\begin{array}{l}n \\ k\end{array}\right)(-1)^{k}(N-n+k)^{j-1}=0, \quad \forall j=1, \cdots, n$.

Proof: By expanding $(N-n+k)^{j-1}, \Lambda(j)$ as defined in (41) can be rewritten as

$$
\Lambda(j)=\sum_{r=0}^{j-1}\left(\begin{array}{c}
j-1 \\
r
\end{array}\right)(N-n)^{j-1-r} g(r)
$$

where $g(r) \triangleq \sum_{k=0}^{n}\left(\begin{array}{l}n \\ k\end{array}\right)(-1)^{k} k^{r}$. We first consider the domain of $r$ such that $g(r)=0$.

Define $f(x)=(x-1)^{n}=\sum_{k=0}^{n}\left(\begin{array}{l}n \\ k\end{array}\right)(-1)^{k} x^{n-k}$. Differentiating $f(x) m$ times, $m=0,1 \cdots n-1$, and noting the two definitions for $f(x)$, we get

$$
\begin{aligned}
f^{m}(x) & =n(n-1) \cdots(n-m+1)(x-1)^{n-m} \\
& =\sum_{k=0}^{n}\left(\begin{array}{l}
n \\
k
\end{array}\right)(-1)^{k} \prod_{p=k}^{k+m}(n-p) x^{n-k-m} .
\end{aligned}
$$

Letting $x=1$ and rewritting $(n-k)(n-k-1) \cdots(n-k-$ $m+1)=\sum_{r=0}^{m} c_{r, m} k^{r}$ for some $c_{j, m}$, we get

$$
f^{m}(1)=\sum_{k=0}^{n}\left(\begin{array}{l}
n \\
k
\end{array}\right)(-1)^{k} \sum_{r=0}^{m} c_{r, m} k^{r}=0
$$

which can be further simplified to

$$
f^{m}(1)=\sum_{r=0}^{m} c_{r, m} g(r)=0 .
$$

Consider $m=0$; we get $f(1)=g(0)=0$. Consider $m=1$; we get $g(1)=0$ since $f^{1}(1)=c_{0,1} g(0)+c_{1,1} g(1)=0$. Thus, from (45), we have by induction for $m=0,1, \cdots n-1$, $g(m)=0, m=0,1, \cdots n-1$.

Now, note from (42) that for $\Lambda(j)=0$, a sufficient condition is that $g(r)=0$ for $r=0, \cdots, j-1$. The largest value of $r$ such that the expression $g(r)=0$ is $n-1$. Hence, we conclude that $\Lambda(j)=0$ for $j=1,2, \cdots n$.

We are now ready to prove Corollary 3.

Proof: Note that 1) the diversity advantage of the lower and upper BER bounds are the same since they only differ in SNR by $10 \log _{\{10\}} 10(M-1) \mathrm{dB}$, and 2$)$ the diversity advantage of the exact BER is also bounded by that of the lower and upper bounds. Thus, it is determined by that of the lower (or upper) bound, which we need to prove to be at least $i$. Using (33), we can rewrite (29) for $L=1$ as $P_{\text {up }}(i, \bar{\gamma})=(M ! / i !(M-i-$ $1) !) \sum_{j=1}^{\infty}\left(a_{j} / \bar{\gamma}_{\text {low }, i}^{j} \Lambda(j)\right.$, where

$$
\Lambda(j) \triangleq \sum_{k=0}^{i}\left(\begin{array}{l}
i \\
k
\end{array}\right)(-1)^{k}(M-i+k)^{j-1} .
$$

Since $\Lambda(j)=0, \forall j=1, \cdots, i$, as shown in Lemma 1 , we have

$$
P_{\text {up }}(i, \bar{\gamma})=\frac{M !}{i !(M-i-1) !} \sum_{j=i}^{\infty} \frac{a_{j}}{\bar{\gamma}_{\text {low }, i}^{j}} \Lambda(j) .
$$

Therefore, $\lim _{\bar{\gamma} \rightarrow \infty}\left(-\log \left(P_{\mathrm{up}}(i, \bar{\gamma})\right) / \log (\bar{\gamma})\right) \geq i+1$, which shows that the diversity advantage is at least $i+1$ for the $i$ th iteration at high SNR.

\section{REFERENCES}

[1] J. Bingham, "Multicarrier modulation for data transmission: an idea whose time has come," IEEE Commun. Mag., vol. 28, no. 5, pp. 5-14, May 1990.

[2] R. van Nee and R. Prasad, OFDM Wireless Multimedia Communications. London, U.K.: Artech House, 2000.

[3] "Radio Broadcasting Systems: Digital Audio Broadcasting (DAB) to Mobile, Portable and Fixed Receivers," ETSI Tech. Rep. ETS 300401 , Feb. 1995.

[4] "Digital Video Broadcasting (DVB); Frame Structure, Channel Coding and Modulation for Digital Terrestrial Television (DVB-T)," ETSI Tech. Rep. ETS 300 744, Mar. 1997.

[5] R. van Nee, G. Awater, M. Morikura, H. Takanashi, M. Webster, and K. Halford, "New high-rate wireless LAN standards," IEEE Commun. Mag., vol. 37, no. 12, pp. 82-88, Dec. 1999.

[6] J. Proakis, Digital Communications, 3rd ed, Singapore: McGraw-Hill, 1995.

[7] K. Fazel, "Performance of CDMA/OFDM for mobile communication systems," in Proc. IEEE Second Int. Conf. Universal Personal Commun., Ottawa, ON, Canada, Oct. 1993, pp. 975-979.

[8] V. Nangia and K. Baum, "Experimental broadband OFDM system: field results for OFDM and OFDM with frequency domain spreading," in Proc. 56th IEEE Veh. Technol. Conf., Vancouver, BC, Canada, Sep. 2002, pp. 223-227.

[9] Z. Dlugaszewski and K. Wesolowski, "WHT/OFDM-an improved OFDM transmission method for selective fading channels," in Proc. Symp. Commun. Veh. Technol., Leuven, Belgium, Oct. 2000, pp. 144-149. 
[10] Z. Lei, Y. Wu, C. K. Ho, S. Sun, P. He, and Y. Li, "Iterative detection for Walsh-Hadamard transformed OFDM," in Proc. 57th IEEE Veh. Technol. Conf., Jeju, Korea, Apr. 2003, pp. 637-640.

[11] N. Yee, J. P. M. G. Linnartz, and G. Fettweis, "Multi-carrier-CDMA in indoor wireless networks," in Proc. IEEE PIMRC, Yokohama, Japan, Sept. 1993, pp. 109-113.

[12] H. Sari, G. Karam, and I. Jeanclaude, "Frequency-domain equalization of mobile radio and terrestrial broadcast channels," in Proc. IEEE Global Telecommun. Conf., San Francisco, CA, Nov. 1994, pp. 1-5.

[13] D. Falconer, S. Ariyavisitakul, A. Benyamin-Seeyar, and B. Eidson, "Frequency domain equalization for single-carrier broadband wireless system," IEEE Commun. Mag., vol. 40, no. 4, pp. 58-66, Apr. 2002.

[14] A. Scaglione, G. B. Giannakis, and S. Barbarossa, "Redundant filterbank precoders and equalizers Part I: unification and optimal designs," IEEE Trans. Signal Process., vol. 47, no. 7, pp. 1988-2006, Jul. 1999.

[15] Y. Ding, T. N. Davidson, Z.-Q. Luo, and K. M. Wong, "Minimum BER block precoders for zero-forcing equalization," IEEE Trans. Signal Process., vol. 51, no. 9, pp. 2410-2423, Sep. 2003.

[16] Y.-P. Lin and S.-M. Phoong, "BER minimized OFDM systems with channel independent precoders," IEEE Trans. Signal Process., vol. 51, no. 9, pp. 2369-2380, Sep. 2003.

[17] Z. Liu, Y. Xin, and G. B. Giannakis, "Space-time-frequency coded OFDM over frequency-selective fading channels," IEEE Trans. Signal Process., vol. 50, no. 10, pp. 2465-2476, Oct. 2002.

[18] A. Bury, J. Egle, and J. Lindner, "Diversity comparison of spreading transforms for multicarrier spread spectrum transmission," IEEE Trans. Commun., vol. 51, no. 5, pp. 774-781, May 2003.

[19] M. K. Varanasi and B. Aazhang, "Multistage detection in asynchronous code-division multiple-access communications," IEEE Trans. Commun., vol. 38, no. 4, pp. 509-519, Apr. 1990.

[20] — - "Near-optimum detection in synchronous code-division multipleaccess systems," IEEE Trans. Commun., vol. 39, no. 5, pp. 725-736, May 1991.

[21] Y. Xin, Z. Wang, and G. B. Giannakis, "Space-time diversity systems based on linear constellation precoding," IEEE Trans. Wireless Commun., vol. 2, no. 2, pp. 294-309, Mar. 2003.

[22] D. A. Wiegandt, Z. Wu, and C. R. Nassar, "High-throughput, high-performance OFDM via pseudo-orthogonal carrier interferometry spreading codes," IEEE Trans. Commun., vol. 51, no. 7, pp. 1123-1134, Jul. 2003.

[23] M. Debbah, W. Hachem, P. Loubaton, and M. de Courville, "MMSE analysis of certain large isometric precoded systems," IEEE Trans. Inf. Theory, vol. 49, no. 5, pp. 1293-1311, May 2003.

[24] M. Park, H. Jun, J. Cho, N. Cho, D. Hong, and C. Kang, "PAPR reduction in OFDM transmission using Hadamard transform," in Proc. IEEE Int. Conf. Commun., New Orleans, LA, Jun. 2000, pp. 430-433.

[25] Y. Wu, C. K. Ho, and S. Sun, "On some properties of Walsh-Hadamard transformed OFDM," in Proc. 56th IEEE Veh. Technol. Conf., Vancouver, BC, Canada, Sep. 2002, pp. 2096-2100.

[26] S. Hara and R. Prasad, "Overview of multicarrier CDMA," IEEE Commun. Mag., vol. 35, no. 12, pp. 126-133, Dec. 1997.

[27] V. Tarokh, H. Jafarkhani, and A. R. Calderbank, "Space-time block codes from orthogonal designs," IEEE Trans. Inf. Theory, vol. 45, no. 5, pp. 1456-1467, May 1999.

[28] M. K. Simon and M.-S. Alouini, Digital Communication Over Fading Channels: A Unified Approach to Performance Analysis. New York: Wiley, 2000.

[29] A. Papoulis, Probability, Random Variables, and Stochastic Processes, 3rd ed. New York: McGraw-Hill, 1991.

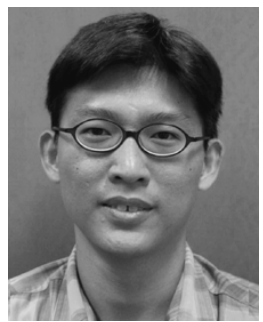

Chin Keong Ho (S'01) received the B.Eng. (firstclass Honors) and M.Eng degrees from the Department of Electrical Engineering, National University of Singapore in 1999 and 2001, respectively. He is working toward the Ph.D. degree at Eindhoven University of Technology, Eindhoven, The Netherlands, conducting joint work with Philips Research.

$\mathrm{He}$ was with the Institute for Infocomm Research, Singapore, from August 2000 to October 2004. His research interest lies in signal processing and wireless communications for multicarrier and space-time communications.

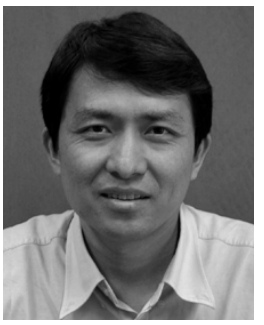

Zhongding Lei (SM'04) received the B.S. degree (Honor) from the Department of Mathematics, Northern Jiaotong University (NJTU, now Beijing Jiaotong University), Beijing, China, in 1991, the M.E. degree from the Department of Telecommunications, Shanghai Institute of Railway Technology (now Tong Ji University), Shanghai, China, in 1994, and the Ph.D. degree from the Institute for Information Science, NJTU, in 1997.

Since 2003, he has been a Scientist in the Digital Wireless Department, Institute for Infocomm Research $\left(I^{2} R\right)$, Singapore. From 2001 to 2003, he was a Member of the Technical Staff in the Institute for Communications Research (ICR, merged into $\mathrm{I}^{2} \mathrm{R}$ ), Singapore. From 1999 to 2001, he was a Staff Research Engineer in the Center for Wireless Communications (CWC, merged into ICR), Singapore. From 1997 to 1999, he was a Post Doctoral Fellow with the Department of Electrical and Computer Engineering, National University of Singapore, and was attached to CWC. His research interests include MIMO, OFDM, UWB radio technology, smart antenna, wireless LAN, and 4G cellular systems. He has published over 40 papers in internationally refereed journals and conferences and has filed three patents on the design of communication systems.

Dr. Lei has been actively serving as a technical reviewer for various IEEE TRANSACTIONS and conferences.

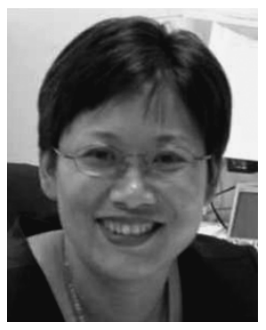

Sumei Sun received the B.Sc. degree from Peking University, Beijing, China, in 1990 and the M.Eng. degree from Nanyang Technological University, Singapore, in 1996. She is working toward the Ph.D. degree on a part-time basis at National University of Singapore.

She worked as an Assistant Lecturer with the Electronics Department, Peking University, from July 1990 to June 1993 and a Research Assistant with the School of Electrical and Electronics Engineering, Nanyang Technological University, from November 1993 to July 1995. Since August 1995, she has been with the Institute for Infocomm Research (previously known as the Centre for Wireless Communications) and involved in R\&D projects on CDMA multiuser detection and interference cancellation, 3G W-CDMA downlink terminal algorithms, and broadband wireless access transceivers. Since 2001, she has been leading the MIMO-OFDM effort for high data rate wireless LAN/PAN applications at $\mathrm{I}^{2} \mathrm{R}$. Her research interests include error correction coding, MIMO system architecture, and iterative receiver algorithms.

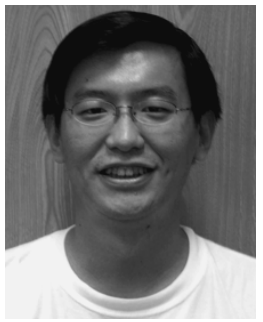

Wu Yan received the B.Eng. (first-class Honors) and M.Eng. degrees from the Department of Electrical Engineering, National University of Singapore, in 1999 and 2001, respectively.

Since 2001, he has been with the Digital Wireless Department, Institute for Infocomm Research, Singapore, where he is currently an Associate Scientist. His research interest lies in the area of signal processing, coding, and modulation for multicarrier and space-time communications. 